\title{
Targeting PLKs as a therapeutic approach to well-differentiated thyroid cancer
}

\author{
Shu-Fu Lin1,2, Jen-Der Lin1,2, Chun-Nan Yeh2,3, Yu-Tung Huang4, Ting-Chao Chou5,† and Richard J Wong6 \\ 1Department of Internal Medicine, Chang Gung Memorial Hospital, Taoyuan, Taiwan \\ ${ }^{2}$ Chang Gung University, Taoyuan, Taiwan \\ ${ }^{3}$ Department of Surgery, Chang Gung Memorial Hospital, Taoyuan, Taiwan \\ ${ }^{4}$ Center for Big Data Analytics and Statistics, Chang Gung Memorial Hospital, Taoyuan, Taiwan \\ 5Laboratory of Preclinical Pharmacology Core, Memorial Sloan-Kettering Cancer Center, New York, New York, USA \\ ${ }^{6}$ Department of Surgery, Memorial Sloan-Kettering Cancer Center, New York, New York, USA \\ Correspondence should be addressed to S-F Lin: mmg@cgmh.org.tw \\ ${ }^{\dagger}($ T-C Chou is now at PD Science, Inc., Paramus, New Jersey, USA)
}

\begin{abstract}
Polo-like kinases (PLKs) are pivotal regulators of cell proliferation and cell survival; therefore, PLKs may be potential targets in the treatment of malignancy. The therapeutic effects of volasertib, a PLKs inhibitor for papillary and follicular thyroid cancer (known as well-differentiated thyroid cancer (WDTC)), were evaluated in this study. Volasertib inhibited cell proliferation in two papillary and two follicular thyroid cancer cell lines in a dose-dependent manner. Volasertib treatment reduced cells in the $\mathrm{S}$ phase and increased cells in the G2/M phase. Volasertib activated caspase-3 activity and induced apoptosis. Drug combinations of volasertib and sorafenib showed mostly synergism in four welldifferentiated thyroid carcinoma cell lines in vitro. Volasertib treatment in vivo retarded the growth of a papillary thyroid tumor model. Furthermore, the combination of volasertib with sorafenib was more effective than a single treatment of either in a follicular thyroid cancer xenograft model. Promising safety profiles appeared in animals treated with either volasertib alone or volasertib and sorafenib combination therapy. These findings support volasertib as a potential drug for the treatment of patients with WDTC.
\end{abstract}

\author{
Key Words \\ - volasertib \\ - polo-like kinase inhibitor \\ - sorafenib \\ - well-differentiated thyroid \\ cancer
}

\section{Introduction}

The incidence of thyroid cancer has increased worldwide over the past four decades (Cabanillas et al. 2016, Kitahara $\&$ Sosa 2016). The transformation of thyroid follicular cells leads to different types of thyroid malignancy, including papillary and follicular thyroid cancer (known as well-differentiated thyroid cancer (WDTC)) and poorly differentiated and anaplastic thyroid cancer (Fagin \& Wells 2016). WDTC accounts for more than $85 \%$ of patients with thyroid carcinoma. Most patients with WDTC survive for more than 10 years after diagnosis following standard treatment with surgery, radioactive iodine (RAI) and thyroid hormone therapy. However, a small proportion of patients who develop metastatic RAI-refractory WDTC have the mean life expectancy of 3-5 years and the 10-year survival rate is only $10 \%$ (Durante et al. 2006). Two multi-kinase inhibitors, sorafenib and lenvatinib, have been approved for the treatment of metastatic and RAI-refractory WDTC by the US Food and Drug Administration (FDA). However, many patients acquire resistance to these drugs or experience toxicities, resulting in dose reduction and termination of treatment (Brose et al. 2014, Schlumberger et al. 2015). 
Novel therapeutic strategies with distinct mechanisms are mandatory to meet the clinical needs of patients with aggressive WDTC.

Polo-like kinases (PLKs) are a family of serine/ threonine kinases that performs multiple cellular functions, including cell cycle progression and cell survival (Strebhardt et al. 2010, Zitouni et al. 2014). There are five human PLK family proteins (PLK1-5) (de Cárcer et al. 2011a). PLK1 regulates centrosome maturation, mitotic progression, DNA damage repair and inhibition of apoptosis (Liu \& Erikson 2003, Yata et al. 2012, Zitouni et al. 2014, Shao et al. 2018). PLK2 and PLK4 are required for centrosome duplication (Habedanck et al. 2005, Cizmecioglu et al. 2012). PLK3 is involved in DNA replication, G1/S and G2/M transition and induction of apoptosis under stress conditions (Zitouni et al. 2014, Helmke et al. 2016). PLK5 is mostly expressed in the brain and may function as a tumor suppressor (de Cárcer et al. 2011b). Given the essential roles of the function of PLKs in cell proliferation and cell survival, PLKs are attractive targets for anticancer therapy, and a variety of PLK inhibitors have been evaluated in the treatment of malignancies (Sebastian et al. 2010, Zitouni et al. 2014, Gjertsen et al. 2015, Kosco et al. 2018).

Volasertib is a potent inhibitor of PLK1, PLK2 and PLK3, with $50 \%$ inhibitory concentrations in the nanomolar range $(0.87 \mathrm{nmol} / \mathrm{L}, 5 \mathrm{nmol} / \mathrm{L}$ and $56 \mathrm{nmol} / \mathrm{L}$, respectively) (Rudolph et al. 2009). Volasertib has been shown to induce G2/M arrest, activate caspase-3 activity and induce apoptosis in vitro (Nguyen et al. 2017). Administration of volasertib has good tissue penetration, a long terminal half-life and potent efficacy in inhibiting tumor growth of colon, lymphoma and leukemia xenografts, with promising safety profiles in animal models (Rudolph et al. 2009, 2015, Nguyen et al. 2017). Recently, single-agent volasertib therapy has revealed encouraging antitumor activity in patients with ovarian cancer in a phase II clinical trial (Pujade-Lauraine et al. 2016). In this study, we sought to evaluate the therapeutic effects of volasertib alone and in combination with sorafenib, a multi-kinase inhibitor approved for the treatment of thyroid cancer, in WDTC in vitro and in vivo.

\section{Materials and methods}

\section{Cell lines}

Two human papillary thyroid cancer cell lines, BHP7-13 and K1, and two human follicular thyroid cancer cell lines, FTC-133 and RO82-W-1, were studied.
BHP7-13 cells were described before (Lin et al. 2017). K1, FTC-133 and RO82-W-1 were obtained from Sigma. All cell lines except RO82-W-1 were authenticated using DNA short tandem repeats (STRs) profiling and stored in liquid nitrogen until use (Schweppe et al. 2008). BHP7-13 and TPC1 are considered genetically identical cell lines because they have identical DNA STR profiling (Schweppe et al. 2008). BHP7-13 cells were maintained in RPMI 1640 with sodium bicarbonate $(2.0 \mathrm{~g} / \mathrm{L})$. K1 and RO82-W-1 cells were maintained in DMEM, Ham's F12 and MCDB 105 (2:1:1) with glutamine $(2.0 \mathrm{mmol} / \mathrm{L})$. FTC-133 cells were maintained in DMEM and Ham's F12 (1:1) with glutamine $(2.0 \mathrm{mmol} / \mathrm{L})$. All media contained $10 \%$ fetal calf serum, 100,000 units/L penicillin and $100 \mathrm{mg} / \mathrm{L}$ streptomycin. All cells were maintained in a $5 \% \mathrm{CO}_{2}$ humidified incubator at $37^{\circ} \mathrm{C}$. The maximum passage number of WDTC cells after thawing was limited to 20 for this study.

\section{Pharmacologic agents}

Volasertib, sorafenib and GSK461364 were obtained from Selleck Chemicals. Volasertib, sorafenib and GSK461364 were dissolved in DMSO (Sigma) to a concentration of $10 \mathrm{mmol} / \mathrm{L}$ and stored at $-80^{\circ} \mathrm{C}$ until further use for in vitro experiments. For the in vivo studies, volasertib was diluted in poly(ethylene glycol) 300 (Sigma) and distilled water $(2: 3 \mathrm{v} / \mathrm{v})$ to a final concentration of $3 \mathrm{mg} / \mathrm{mL}$ and stored at $-80^{\circ} \mathrm{C}$ until use. Sorafenib was dissolved in $50 \%$ Kolliphor EL (Sigma) and 50\% ethanol (Sigma) to a concentration of $57.6 \mathrm{mg} / \mathrm{mL}$ and stored at $-80^{\circ} \mathrm{C}$. Sorafenib was further diluted with water to a final concentration of $14.4 \mathrm{mg} / \mathrm{mL}$ before in vivo use.

\section{Antibodies}

Antibodies targeting cleaved caspase-3, proliferating cell nuclear antigen (PCNA), p-Histone H3 (Ser10), PLK1, PLK2 and PLK3 were purchased from Cell Signaling Technology. $\alpha$-Tubulin and $\beta$-actin antibodies were obtained from Sigma.

\section{Cytotoxicity assays and drug synergy studies}

Cells were plated at $2 \times 10^{3}$ (BHP7-13 and FTC-133) and $2 \times 10^{4}$ cells (K1 and RO82-W-1) per well in 24-well plates in $1 \mathrm{~mL}$ of media. After overnight incubation, six serial two-fold dilutions of volasertib, sorafenib or vehicle were added over a 4-day treatment course after which cytotoxicity was determined. Culture medium was removed, and the cells were washed with PBS and lysed 
with Triton X-100 (1.35\%, Sigma) to release intracellular lactate dehydrogenase (LDH), which was quantified with a Cytotox 96 kit (Promega) at $490 \mathrm{~nm}$ by spectrophotometry (Infinite M200 PRO, Tecan). Each experiment was performed in triplicate, and the results are shown as the percentage of surviving cells determined by comparing the LDH of each sample relative to control samples, which were considered $100 \%$ viable. The median-effect dose $\left(\mathrm{IC}_{50}\right)$ on day 4 was calculated for each cell line using CompuSyn software (Chou \& Martin 2005, Chou 2006).

For combination therapy studies, cells were treated with volasertib and sorafenib at a fixed dose ratio. Cells were incubated with vehicle, volasertib, sorafenib or combination therapy simultaneously for a 4-day course after which cytotoxicity was measured. Interactions between volasertib and sorafenib in vitro were assessed by calculating the combination index (CI) using the Chou-Talalay equation and CompuSyn software. Synergy $(\mathrm{CI}<1)$, additive effect $(\mathrm{CI}=1)$ and antagonism $(\mathrm{CI}>1)$ are quantitatively determined by CompuSyn simulation at different effect levels.

\section{Cell cycle assessment}

The effects of volasertib on cell cycle progression were evaluated. Cells were plated at $4 \times 10^{5}$ cells per well in six-well plates in $2 \mathrm{~mL}$ of media overnight. Volasertib $(100 \mathrm{nmol} / \mathrm{L})$ or vehicle was added and incubated for $24 \mathrm{~h}$, after which adherent cells were collected, washed with PBS, fixed with cold $70 \%$ ethanol and incubated with RNase A $(100 \mu \mathrm{g} / \mathrm{mL}$; Sigma) and propidium iodide ( $5 \mu \mathrm{g} / \mathrm{mL}$; Sigma) at $37^{\circ} \mathrm{C}$ for $15 \mathrm{~min}$. Cell cycle distribution was assessed by DNA content detected by flow cytometry (BD FACScalibur Flow Cytometer, BD Biosciences). Each condition was performed in triplicate.

\section{Apoptosis assessment}

Caspase-3 activity was analyzed using a fluorometric assay kit (Abcam). Cells were plated at $1 \times 10^{6}$ cells in $100-\mathrm{mm}$ Petri dishes in $10 \mathrm{~mL}$ of media overnight. Volasertib $(100 \mathrm{nmol} / \mathrm{L})$ or vehicle was added for $24 \mathrm{~h}$. Adherent cells $\left(5 \times 10^{5}\right)$ were collected, centrifuged, and lysed using $50 \mu \mathrm{L}$ of lysis buffer on ice for $10 \mathrm{~min}$, and incubated with DEVD-AFC substrate and reaction buffer at $37^{\circ} \mathrm{C}$ for $1.5 \mathrm{~h}$. Caspase-3 activity was detected by spectrophotometry. Each condition was performed in duplicate.

The ability of volasertib to induce sub-G1 apoptotic cell accumulation was studied using flow cytometry.
Cells were plated at $2 \times 10^{5}$ (BHP7-13) or $4 \times 10^{5}$ (K1, FTC-133, RO82-W-1) cells per well in six-well plates in $2 \mathrm{~mL}$ media overnight. Volasertib $(100 \mathrm{nmol} / \mathrm{L})$ or vehicle was added and incubated for $24 \mathrm{~h}$. Floating cells and trypsinized adherent cells were collected, and samples were prepared as described above for cell cycle assessment. Apoptotic sub-G1 cells were assessed by DNA content detected by flow cytometry (BD FACScalibur Flow Cytometer, BD Biosciences). Each condition was performed in triplicate.

\section{Immunofluorescence microscopy}

The expression of cleaved caspase- 3 was evaluated using immunofluorescence microscopy. Thyroid cancer cells were plated at $5 \times 10^{4}$ cells in four-well culture slides in $1 \mathrm{~mL}$ of media overnight. Cells were treated with volasertib $(100 \mathrm{nmol} / \mathrm{L})$ or placebo for $24 \mathrm{~h}$, washed with PBS, fixed in $4 \%$ paraformaldehyde (Sigma) for $15 \mathrm{~min}$ at room temperature, washed with PBS, permeabilized with $0.1 \%$ Triton X-100 (10 min, room temperature) and washed with PBS. Cells were then incubated with primary rabbit cleaved caspase- 3 antibody (1:400) and mouse $\alpha$-tubulin antibody (1:1000) at $4^{\circ} \mathrm{C}$ overnight, washed with PBS and incubated with secondary Alexa Fluor 633-conjugated goat anti-rabbit antibody (1:1000; Invitrogen) and Alexa Fluor 488-conjugated goat anti-mouse antibody (1:1000; Life Technologies) for $25 \mathrm{~min}$ at $37^{\circ} \mathrm{C}$, washed with PBS, incubated with $4^{\prime}$,6-diamidino-2-phenylindole (DAPI; $0.2 \mu \mathrm{g} / \mathrm{mL}$, Invitrogen) for $10 \mathrm{~min}$ at room temperature, washed with PBS and covered with mounting medium. Images were acquired using Leica TCS SP8 X confocal microscopy (Leica Microsystems).

The effect of volasertib on mitosis was evaluated using confocal microscopy. Thyroid cancer cells were plated at $1 \times 10^{5}$ cells in four-well culture slides in $1 \mathrm{~mL}$ of media overnight. Volasertib $(100 \mathrm{nmol} / \mathrm{L})$ or placebotreated thyroid cancer cell samples were prepared as described earlier. Cells were then incubated with primary rabbit p-Histone H3 (Ser10) (1:200) and mouse $\alpha$-tubulin antibody (1:1000) at $4^{\circ} \mathrm{C}$ overnight, washed with PBS, incubated with secondary Alexa Fluor 633-conjugated goat anti-rabbit antibody (1:1000) and Alexa Fluor 488-conjugated goat anti-mouse antibody (1:1000) for $25 \mathrm{~min}$ at $37^{\circ} \mathrm{C}$; then samples were prepared as described earlier. Images were captured with Leica TCS SP8 X confocal microscopy (Leica Microsystems). Chromosomes were examined to identify mitotic cells. 


\section{Flank xenograft tumor therapy}

K1 and FTC-133 flank tumors were established by injecting $1 \times 10^{6}$ cells in $100 \mu \mathrm{L}$ of extracellular matrix (ECM) gel (Sigma) into the subcutaneous flanks of female athymic nude mice 10-11 weeks of age from the National Laboratory Animal Center, Taiwan. K1 and FTC-133 cell lines were chosen because they had high tumorigenesis rates in murine models.

For monotherapy with volasertib, mice bearing K1 xenograft tumors received oral administration of vehicle or volasertib $(25 \mathrm{mg} / \mathrm{kg}$ and $30 \mathrm{mg} / \mathrm{kg})$ once a day for two cycles of 2-day on and 5-day off therapy. For volasertib and sorafenib combination oral therapy, mice bearing $\mathrm{K} 1$ and FTC-133 thyroid tumors received placebo, volasertib $(25 \mathrm{mg} / \mathrm{kg})$ once a day of 2-day on and 5-day off therapy, sorafenib $(25 \mathrm{mg} / \mathrm{kg})$ once a day of 4-day on and 3-day off therapy or combination treatment for three cycles of therapy. Tumor dimensions were serially measured with electronic calipers, and the volumes were calculated by the following formula: $a \times b^{2} \times 0.4$, where $a$ represents the largest diameter and $b$ is the perpendicular diameter. The body weight of each animal was followed as a marker of toxicity. Relative tumor growth of each xenograft was calculated as $V x / V 1$, where $V x$ is the volume in $\mathrm{mm}^{3}$ at an indicated time and $V 1$ at the beginning of treatment.

Tumor levels of PCNA (a marker for cell proliferation) (Moldovan et al. 2007) and cleaved caspase-3 were evaluated in mice treated with oral dosing of volasertib ( $25 \mathrm{mg} / \mathrm{kg}$ ) by Western blot analysis. At indicated periods, animals were killed with carbon dioxide, and the tumors were harvested, mixed with protein extraction buffer (GE Healthcare), homogenized and sonicated on ice. After centrifugation, clarified supernatants were aliquoted and stored at $-80^{\circ} \mathrm{C}$ until Western blot analyses.

This study was approved by the Committee of Laboratory Animal Center at the Chang Gung Memorial Hospital, Linkou (permission No: 2013121401) and performed in accordance with the recommendations in the Guide for the Care and Use of Laboratory Animals of the Chang Gung Memorial Hospital.

\section{Western blot analysis}

Thyroid tumor samples were collected and prepared as described earlier. Total protein $(20-40 \mu \mathrm{g})$ was separated by electrophoresis on $12 \%$ Tris- $\mathrm{HCl}$ gels, transferred to PVDF membranes and blocked and exposed to primary antibodies followed by a secondary antibody conjugated to horseradish peroxidase. Signals were developed using an enhanced chemiluminescence kit (PerkinElmer and Merck Millipore).

\section{Statistical analyses}

Statistical analyses were performed using IBM SPSS software (version 25). Kolmogorov-Smirnov test was used for assessment of normality. Student's $t$ test was carried out to analyze in vitro data. In vivo data were analyzed by two-way ANOVA with post hoc Scheffe test. Pearson's correlation coefficient was used to measure the association between $\mathrm{IC}_{50}$ of volasertib and the expression of PLK1, PLK2 and PLK3 in four WDTC cell lines. Results are expressed as mean \pm s.e. $P<0.05$ was considered statistically significant.

\section{Results}

\section{Cytotoxicity of volasertib in WDTC cell lines}

Volasertib inhibited cell survival in two papillary thyroid cancer cell lines (BHP7-13 and K1 cells) and two follicular thyroid cancer cell lines (FTC-133 and RO82-W-1 cells) in a dose-dependent manner (Fig. 1A). Volasertib at $100 \mathrm{nmol} / \mathrm{L}$ arrested cell growth by $99.1 \%$ (BHP7-13), $76.0 \%$ (K1), $77.5 \%$ (FTC-133) and $57.7 \%$ (RO82-W-1) on day 4 . The cytotoxicity potency of volasertib in WDTC cell lines was determined using CompuSyn software. The median-effect dose $\left(\mathrm{IC}_{50}\right)$ was determined on day 4 (Fig. 1B). In papillary thyroid cancer cell lines, BHP7-13 cells had a lower $\mathrm{IC}_{50}(18.1 \pm 0.0 \mathrm{nmol} / \mathrm{L})$ than that of $\mathrm{K} 1$ cells $(59.8 \pm 0.6 \mathrm{nmol} / \mathrm{L})$. In follicular thyroid cancer cell lines, FTC-133 cells had a lower IC $_{50}(21.6 \pm 0.8 \mathrm{nmol} / \mathrm{L})$ than that of RO82-W-1 cells $(71.5 \pm 2.7 \mathrm{nmol} / \mathrm{L})$.

\section{Effects of volasertib on the cell cycle}

The effect of volasertib ( $100 \mathrm{nmol} / \mathrm{L}$ for $24 \mathrm{~h})$ on cell cycle distribution in papillary and follicular thyroid cancer cell lines was evaluated (Supplementary Fig. 1, see section on supplementary data given at the end of this article), and the cell cycle data were analyzed (Fig. 2A). Compared with placebo treatment, volasertib significantly induced cell accumulation in the $\mathrm{G} 2 / \mathrm{M}$ phase as compared with control treatment in BHP7-13 $(64.2 \pm 0.5 \%$ and $8.5 \pm 0.1 \%$, $P<0.001), \quad \mathrm{K} 1 \quad(22.1 \pm 0.2 \%$ and $10.2 \pm 0.1 \%, \quad P<0.001)$, FTC-133 $(82.1 \pm 0.3 \%$ and $12.2 \pm 0.1 \%, P<0.001)$ and RO82-W-1 cells $(85.5 \pm 0.3 \%$ and $13.9 \pm 0.1 \%, P<0.001)$. Besides, volasertib significantly decreased cells in the $S$ (c) 2019 Society for Endocrinology Published by Bioscientifica Ltd. Printed in Great Britain 


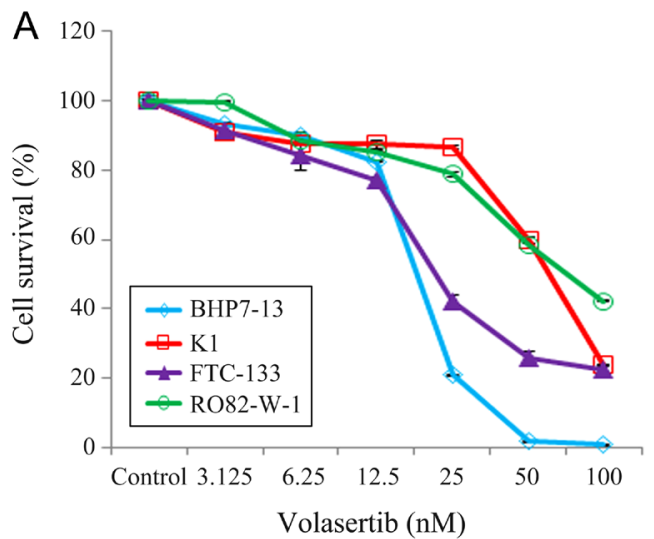

phase in BHP7-13 $(3.8 \pm 0.3 \%$ and $8.9 \pm 0.2 \%, P<0.001)$, K1 $(10.5 \pm 0.0 \%$ and $12.7 \pm 0.2 \%, \quad P<0.001), \quad$ FTC-133 $(4.1 \pm 0.1 \%$ and $5.6 \pm 0.2 \%, P=0.002)$ and RO82-W-1 cells $(4.7 \pm 0.2 \%$ and $8.1 \pm 0.1 \%, P<0.001)$.

The ability of volasertib to arrest cells in the mitotic phase was determined using confocal microscopy. A representative cell line, BHP7-13 cells, is shown (Supplementary Fig. 2). Mitotic cells were identified and the mitotic index was calculated for papillary and follicular thyroid cancer cell lines (Fig. 2B). Compared with the control treatment, volasertib $(100 \mathrm{nmol} / \mathrm{L})$ treatment for $24 \mathrm{~h}$ significantly increased the percentage of mitotic cells in BHP7-13 (7.9 $\pm 1.5 \%$ and $2.4 \pm 0.2 \%, P=0.002), \mathrm{K} 1$ $(3.6 \pm 0.4 \%$ and $1.2 \pm 0.2 \%, P<0.001)$, FTC $-133(5.2 \pm 0.5 \%$ and $2.6 \pm 0.3 \%, P<0.001)$ and RO82-W-1 cells $(9.6 \pm 0.9 \%$ and $1.6 \pm 0.2 \%, P<0.001)$, demonstrating that volasertib accumulated thyroid cancer cells in mitosis.

The distribution of cells in mitosis was evaluated (Fig. 2C). Compared with control treatment, volasertib $(100 \mathrm{nmol} / \mathrm{L}$ for $24 \mathrm{~h})$ significantly increased the percentage of prometaphase cells in BHP7-13, K1, FTC-133 and RO82-W-1. This finding of mitotic arrest in prometaphase is a characteristic phenotype of PLK arrest.

\section{Volasertib-induced apoptosis in WDTC cell lines}

Induction of apoptosis has an important role in cancer therapy (Pfeffer \& Singh 2018). Volasertib activates
A

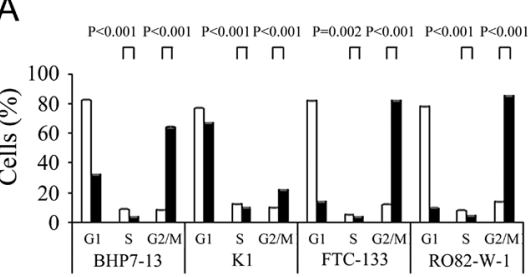

C

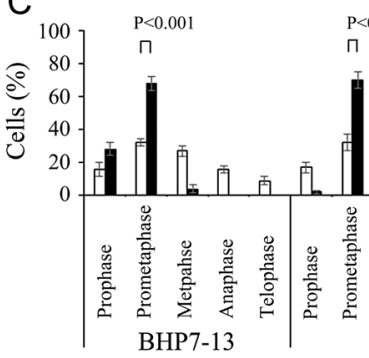

Control, $\mathrm{n}=\quad 100$

Volasertib, $\mathrm{n}=100$

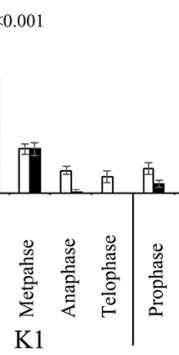

K1

100
100

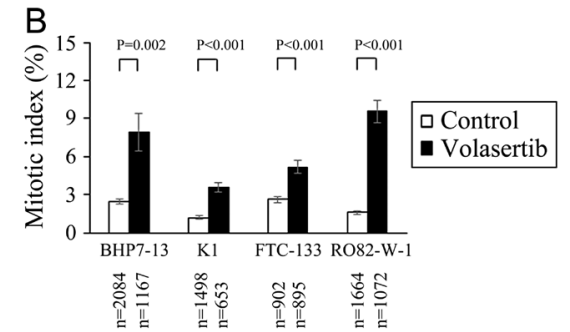

0.001

FT

$$
\Pi
$$

$\mathrm{P}<0.001$

$\square$ Control

- Volasertib

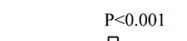

Figure 2

Volasertib decreases cells in the S phase, accumulates cells in the G2/M phase and inhibits mitotic progression in prometaphase in well-differentiated thyroid cancer cells. (A) Cell cycle distribution was analyzed by evaluating the DNA content in well-differentiated thyroid cancer cells treated with placebo or volasertib $(100 \mathrm{nmol} / \mathrm{L})$ for $24 \mathrm{~h}$ using flow cytometry. Volasertib treatment significantly reduced cells in the $S$ phase and accumulated cells in the G2/M phase in four cell lines. (B) The proportion of well-differentiated thyroid cancer cells in mitosis was assessed after treatment with volasertib (100 nmol/L) or placebo for $24 \mathrm{~h}$. Cells were stained with DAPI, and chromosome characteristics were evaluated using immunofluorescence confocal microscopy. The mitotic index was assessed with a minimum of 653 cells counted from 10 different fields for each condition. Volasertib significantly increased the proportion of BHP7-13, K1, FTC-133 and RO82-W-1 cells in mitosis. (C) The distribution of cells in mitosis was determined by counting 100 mitotic cells by confocal microscopy for each condition. Quantification analyses revealed mitotic cells were arrested in prometaphase by the treatment of volasertib $(100 \mathrm{nmol} / \mathrm{L})$ for $24 \mathrm{~h}$. 
caspase-3 activity and induces apoptosis in lymphoma cells (Nguyen et al. 2017). Therefore, we evaluated the effects of volasertib on apoptosis in papillary and follicular thyroid cancer cell lines. The effects of volasertib $(100 \mathrm{nmol} / \mathrm{L})$ on caspase- 3 activity in BHP7-13, K1, FTC-133 and RO82-W-1 cell lines were determined using a fluorometric assay at $24 \mathrm{~h}$ (Fig. 3A). Volasertib significantly increased caspase-3 activity compared to control treatment in BHP7-13, K1, FTC-133 and RO82-W-1 cells, demonstrating activation of caspase-3. Caspase- 3 activation was also assessed by detection of cleaved caspase- 3 using immunofluorescent analysis in papillary and follicular thyroid cancer cell lines (Fig. 3B). The percentages of cells with cleaved caspase-3 (active form of caspase-3) expression were analyzed (Fig. 3C). Volasertib $(100 \mathrm{nmol} / \mathrm{L}$ for $24 \mathrm{~h})$ significantly increased the proportions of cells with cleaved caspase- 3 expression in BHP7-13, K1, FTC-133 and RO82-W-1 cells when compared with the corresponding controls. Therefore, activation of caspase- 3 may lead to apoptotic cell death.

The ability of volasertib to induce sub-G1 apoptosis in papillary and follicular thyroid cancer cell lines was evaluated (Supplementary Fig. 3). BHP7-13, K1, FTC-133 and RO82-W-1 cell lines were exposed to volasertib
$(100 \mathrm{nmol} / \mathrm{L}$ for $24 \mathrm{~h})$, and the proportion of sub-G1 apoptotic cells was calculated (Fig. 3D). Volasertib significantly induced higher proportions of sub-G1 cells in BHP7-13 $(1.3 \pm 0.0 \%$ and $0.5 \pm 0.1 \%, P=0.001), \mathrm{K} 1$ $(7.9 \pm 0.2 \%$ and $5.1 \pm 0.1 \%, P<0.001)$, FTC- $133(9.1 \pm 0.2 \%$ and $5.2 \pm 0.2 \%, P<0.001)$ and RO82-W-1 cells $(1.7 \pm 0.1 \%$ and $1.0 \pm 0.0 \%, P=0.004)$ as compared to the control treatment. These data indicate that volasertib induces apoptosis in papillary and follicular thyroid cancer cells.

\section{Monotherapy with volasertib for murine flank WDTC tumors}

Female nude mice bearing flank xenografts of K1 cells were used to evaluate the therapeutic efficacy and safety of volasertib in papillary thyroid cancer in vivo. Animals with established $\mathrm{K} 1$ flank tumors with a mean diameter of $4.8 \mathrm{~mm}$ were treated with oral gavage of placebo $(n=6)$, low-dose volasertib $(25 \mathrm{mg} / \mathrm{kg}, n=6)$ or high-dose volasertib $(30 \mathrm{mg} / \mathrm{kg}, n=6)$ once a day for two cycles of a 2-day on and 5-day off therapy (Fig. 4A). Low-dose and high-dose volasertib treatment significantly retarded K1 tumor growth as compared to the control group $(P<0.001$ and $P<0.001$, respectively). Serial administration of
A
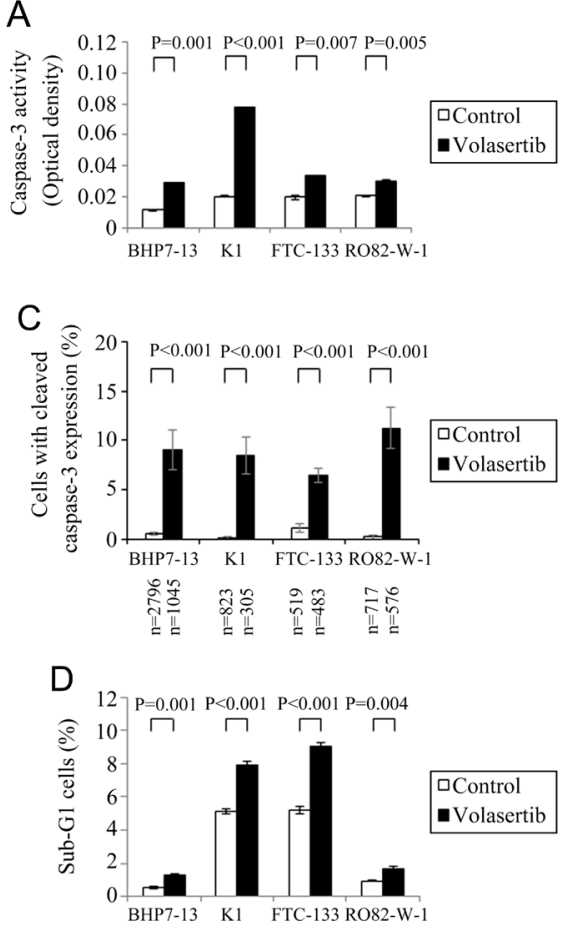

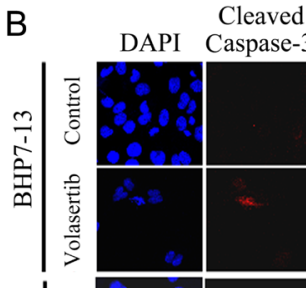

DAPI Cleaved

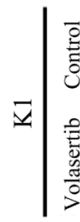
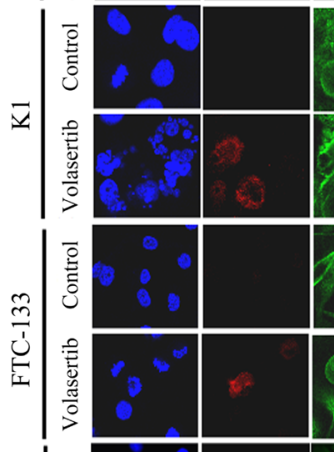

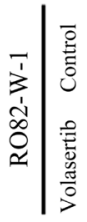

Figure 3

Volasertib stimulates caspase- 3 activity and induces apoptosis in well-differentiated thyroid cancer cells. (A) Caspase-3 activity was evaluated using a fluorometric assay kit in BHP7-13, K1, FTC-133 and RO82-W-1 cells treated with Volasertib (100 nmol/L) or vehicle for $24 \mathrm{~h}$. (B) Well-differentiated thyroid cancer cells were treated with volasertib $(100 \mathrm{nmol} / \mathrm{L})$ or placebo for $24 \mathrm{~h}$ and stained with DAPI (blue) and fluorescent antibodies against cleaved caspase-3 (red) and $\alpha$-tubulin (green). Cells with cleaved caspase-3 expression are shown (arrowhead). (C) The percentages of cells with cleaved caspase-3 expression were assessed after treatment with placebo or volasertib (100 nmol/L) for $24 \mathrm{~h}$. Cells were stained with fluorescent antibodies against cleaved caspase-3 and its expression was evaluated using immunofluorescence confocal microscopy. A minimum of 305 cells from at least 10 different fields were counted for each condition. Volasertib significantly increased the proportion of BHP7-13, K1, FTC-133 and RO82-W-1 cells with cleaved caspase-3 expression. (D) Sub-G1 apoptotic cells were detected by measuring the DNA content of $1 \times 10^{4}$ events for each sample using flow cytometry in cells treated with volasertib $(100 \mathrm{nmol} / \mathrm{L})$ or vehicle for $24 \mathrm{~h}$. Volasertib increased the proportion of sub-G1 cells in four welldifferentiated thyroid cancer cell lines. Scale bar, $20 \mu \mathrm{m}$. 


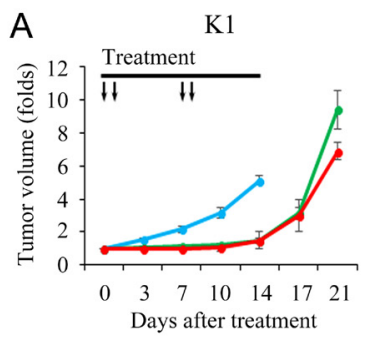

C

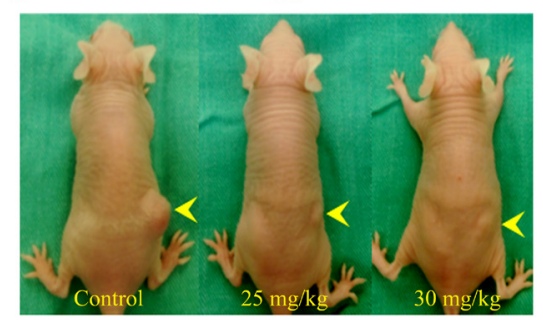

B $\quad \mathrm{K} 1$

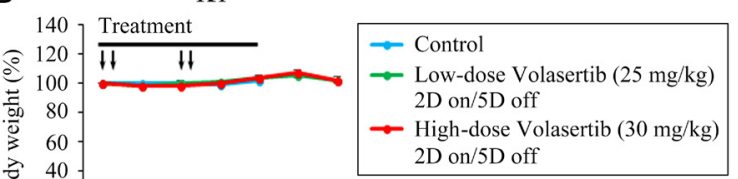

$\mathrm{K} 1$

$D$

Volasertib $(25 \mathrm{mg} / \mathrm{kg}) \quad \boldsymbol{\downarrow}$

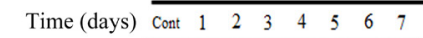

PCNA

Cleaved caspase- 3

$\alpha$-tubulin

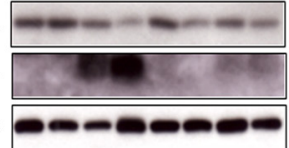

low- and high-dose volasertib did not result in significant body weight change during the study period (Fig. 4B). We did not observe any morbidity in these animals. Representative mice bearing K1 tumors (Fig. 4C) were photographed after a 14-day treatment.

The molecular effects of low-dose volasertib $(25 \mathrm{mg} / \mathrm{kg})$ treatment in K1 xenografts were evaluated using Western blot analysis (Fig. 4D). Volasertib treatment increased the levels of cleaved caspase- 3 on days 2 and 3. The PCNA level was decreased by day 3 .

\section{Interaction of volasertib and sorafenib in WDTC cells}

Interactions between volasertib and sorafenib were studied in papillary and follicular thyroid cancer cell lines (Fig. 5A). Six serial two-fold dilutions were examined at the following starting doses for BHP7-13, K1, FTC-133 and RO82-W-1, respectively: volasertib at $72.4 \mathrm{nmol} / \mathrm{L}$, $239.2 \mathrm{nmol} / \mathrm{L}, \quad 86.4 \mathrm{nmol} / \mathrm{L}$ and $286.0 \mathrm{nmol} / \mathrm{L}$ and sorafenib at $2.8 \mathrm{mmol} / \mathrm{L}, \quad 18.0 \mathrm{mmol} / \mathrm{L}, \quad 23.6 \mathrm{mmol} / \mathrm{L}$ and $31.2 \mathrm{mmol} / \mathrm{L}$. The starting doses of volasertib and sorafenib were four-fold $\mathrm{IC}_{50}$ of each drug for each cell line determined in this and previous studies (Fig. 1 and Supplementary Fig. 4, Lin et al. 2018). In BHP7-13, K1 and FTC-133 cells, the combination of volasertib and sorafenib had significantly improved cytotoxicity over single agent therapies across all doses of treatment. In RO82-W-1 cells, volasertib and sorafenib combination therapy significantly improved cytotoxicity over singleagent therapies at lower doses of treatment. The CI of volasertib and sorafenib was analyzed using the ChouTalalay method, which revealed that the combination of volasertib and sorafenib ranged from synergistic to antagonist in BHP7-13 cells (CI: 0.83-1.80), K1 cells (CI: $0.65-1.08)$ and RO82-W-1 cells (CI: 0.60-1.31) and synergistic in FTC-133 cells (CI: 0.41-0.62; Fig. 5B). These data demonstrated that combination therapy of volasertib and sorafenib was mostly synergistic in BHP7-13, K1, FTC-133 and RO82-W-1 cell lines.

\section{Combination therapy of volasertib and sorafenib for murine flank thyroid tumors}

We sought to evaluate the effect of volasertib and sorafenib combination therapy in mice bearing K1 and FTC-133 xenografts. Animals with established K1 flank tumors with a mean diameter of $4.3 \mathrm{~mm}$ were treated with oral gavage of placebo, volasertib, sorafenib or combination therapy ( $n=6$ per group) once a day for three cycles of therapy (Fig. 6A). The dose of sorafenib was chosen based on a previous report (Lin et al. 2018). Volasertib, sorafenib and combination treatment significantly inhibited K1 tumor growth as compared with control treatment (Fig. 6A). The difference in inhibitory effect between volasertib alone and combination therapy did not achieve statistical significance $(P=0.911)$. Serial treatment of volasertib, sorafenib and combination therapy did not significantly result in decrease in body weight as compared with control treatment (Fig. 6B).

Animals with established FTC-133 flank tumors with a mean diameter of $5.5 \mathrm{~mm}$ were treated with oral gavage of placebo $(n=6)$, volasertib $(n=6)$, sorafenib $(n=5)$ or combination therapy $(n=5)$ once a day for three cycles of therapy (Fig. 6C). Single volasertib and sorafenib did not significantly retard FTC-133 tumor growth as compared with control treatment. However, combination of volasertib 
A

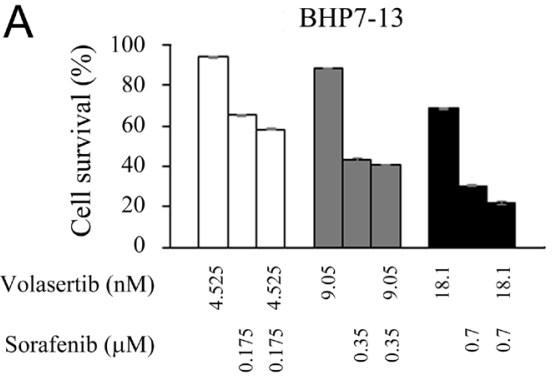

FTC-133
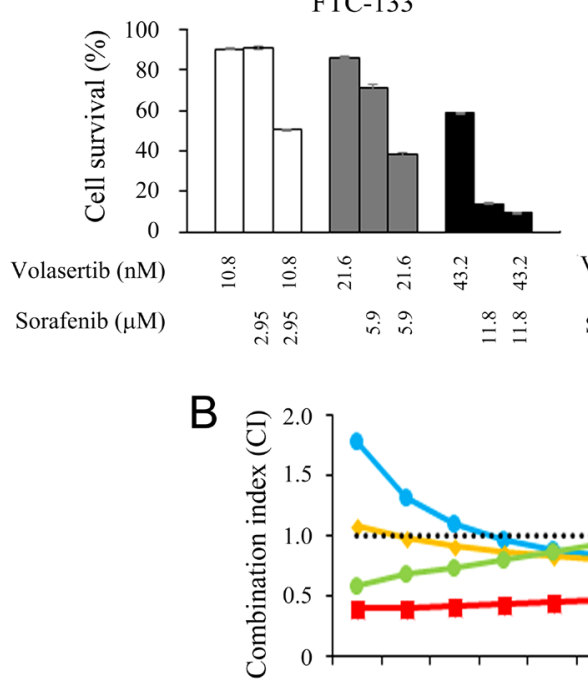

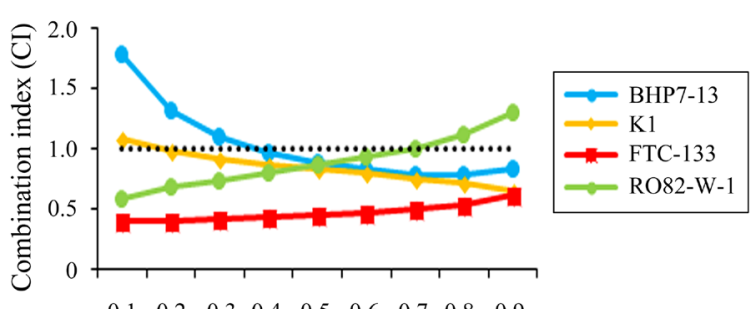

$\begin{array}{lllllllll}0.1 & 0.2 & 0.3 & 0.4 & 0.5 & 0.6 & 0.7 & 0.8 & 0.9\end{array}$

Fraction affected $(\mathrm{Fa})$

K1

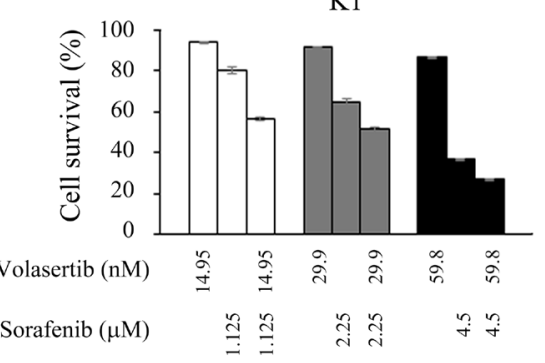

R082-W-1

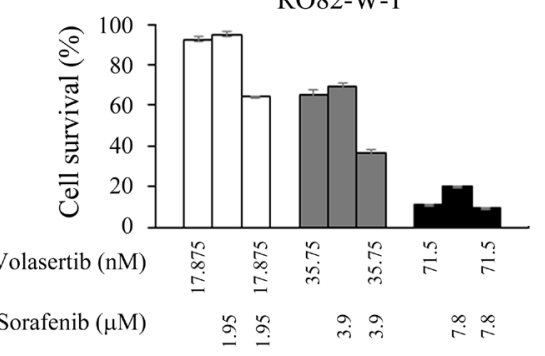

Figure 5

Combination therapy of volasertib and sorafenib in well-differentiated thyroid cancer cells. (A) The cytotoxic effects of volasertib and sorafenib alone and in combination after a 4-day treatment in BHP7-13, K1, FTC-133 and RO82-W-1 cells were evaluated using LDH assays. (B) The combination index $(\mathrm{Cl})$ of volasertib and sorafenib was calculated using CompuSyn software. The combination therapy of volasertib and sorafenib had synergistic effects in FTC-133 cells and synergistic to antagonistic effects in BHP7-13, K1, and RO82-W-1 cells. Volasertib plus sorafenib appeared to have synergistic effects at most conditions in BHP7-13, K1, and RO82-W-1 cell lines. The horizontal line at $\mathrm{Cl}=1$ was drawn for discrimination of synergism $(\mathrm{Cl}<1)$ and antagonism $(\mathrm{Cl}>1)$. Fraction affected $(\mathrm{Fa})$ means the proportion of cell inhibition (percent inhibition/100). and sorafenib treatment significantly inhibited FTC-133 tumor growth as compared with volasertib, sorafenib and placebo therapy. Of note, the combination therapy led to tumor volume reduction in two out of five animals between days 14 and 35, demonstrating that the combination of these two drugs was able to reduce the tumor mass in $40 \%$ of FTC-133 xenografts. Serial treatment of volasertib, sorafenib or combination therapy did not result in substantial changes in body weight as compared with control treatment (Fig. 6D). Representative mice bearing FTC-133 tumors were photographed after 21- and 41-day treatment (Fig. 6E). The PCNA levels decreased and cleaved caspase-3 levels increased with volasertib treatment in FTC-133 xenografts (Supplementary Fig. 5). Volasertib treatment has demonstrated to increase PLK1 expression in HeLa cells (Raab et al. 2015). We observed similar effects of volasertib to increase PLK1 levels in WDTC in vitro (Supplementary Fig. 6A) and in vivo (Supplementary Fig. 6B).

\section{Discussion}

Volasertib effectively inhibited cell survival in papillary and follicular thyroid cancer cell lines. Volasertib treatment alone and in combination with sorafenib effectively inhibited tumor growth of WDTC xenograft models with promising safety profiles. These favorable results suggest that volasertib has the potential for clinical application in the treatment of patients with WDTC.

Volasertib inhibited mitotic progression at prometaphase, which is likely one of the mechanisms of cytotoxicity in the treatment of thyroid cancer cells. Besides mitotic arrest, we also noted that volasertib accumulated cells in G2 phase for BHP7-13, K1, FTC-133 and RO82-W-1 cell lines. Volasertib increased the proportion of $\mathrm{G} 2 / \mathrm{M}$ phase cells over that of $\mathrm{M}$ phase cells, indicating that volasertib arrests cells in G2 phase. Therefore, G2 phase block is likely a key mechanism of cytotoxicity in the treatment of thyroid cancer cells with volasertib. The inhibition of PLK1 may account for G2 arrest and mitotic block. The main PLK1 functions start in G2 and are essential for mitotic entry and mitotic progression (Strebhardt \& Ullrich 2006, Zitouni et al. 2014). The effects of PLK1 inhibition may range from a failure of $\mathrm{G} 2 / \mathrm{M}$ transition to mitotic arrest. In this study, volasertib consistently inhibited G2/M transition and induced mitotic block in the second mitotic phase
C) 2019 Society for Endocrinology Published by Bioscientifica Ltd. Printed in Great Britain 
A

K1

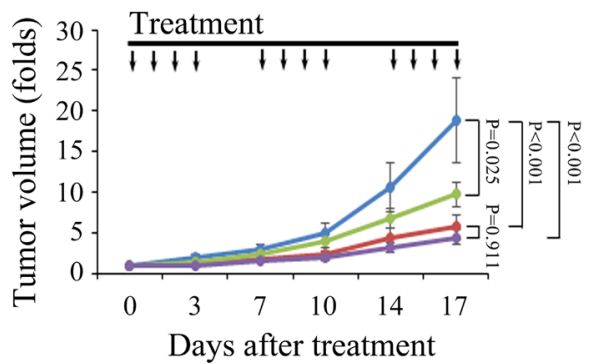

C

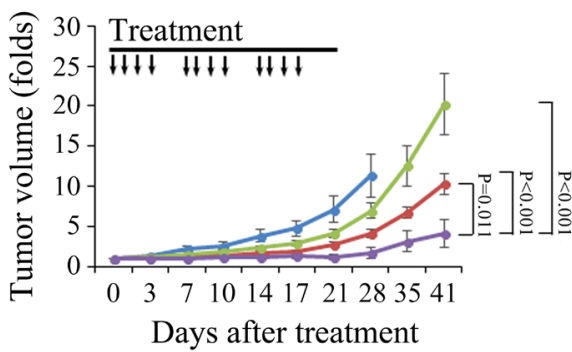

B

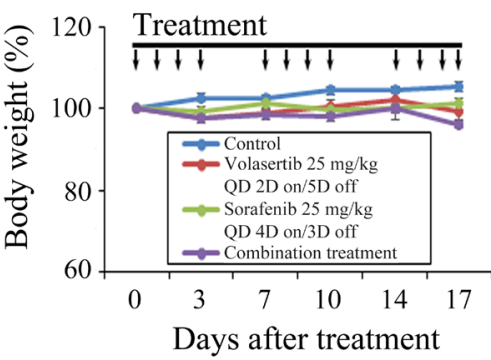

D

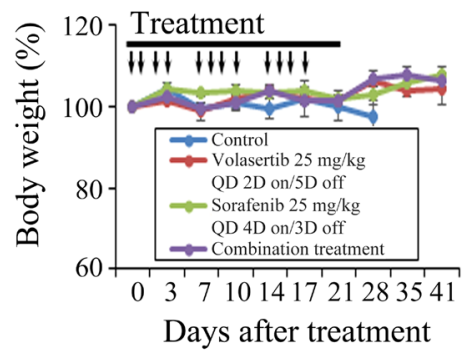

\section{Figure 6}

Therapeutic effects of volasertib and sorafenib in murine $\mathrm{K} 1$ and FTC-133 xenograft tumor models. (A) After K1 flank tumors were established in nude mice, mice were treated with oral gavage of placebo, volasertib ( $25 \mathrm{mg} / \mathrm{kg}$, 2-day on and 5-day off), sorafenib (25 mg/kg, 4-day on and 3-day off) or combination therapy once a day for three cycles of therapy. Compared with control therapy, volasertib, sorafenib and combination treatment significantly inhibited tumor growth. Combination therapy did not significantly retard xenograft growth when compared with volasertib or sorafenib single modality treatment. (B) Volasertib, sorafenib and combination therapy did not significantly decrease body weight as compared with control mice. (C) After FTC-133 flank tumors were established in nude mice, they were treated with oral gavage of placebo, volasertib (25 mg/kg, 2-day on and 5-day off), sorafenib ( $25 \mathrm{mg} / \mathrm{kg}$, 4-day on and 3-day off) or combination therapy for three cycles of therapy. Compared with control therapy, volasertib and sorafenib treatment did not significantly inhibit tumor growth. However, combination therapy significantly retarded xenograft growth when compared with either single agent or control treatment. (D) No substantial decreases in body weight were attributable to volasertib, sorafenib, or combination therapy compared with the control mice. (E) Mice bearing FTC-133 tumors (arrowhead) were photographed on days 21 and 41. Arrow, placebo, volasertib, sorafenib and combination treatment. (prometaphase) in four cell lines. We found GSK461364, a selective PLK1 inhibitor, also led to G2 arrest and mitotic block in WDTC cell lines (Supplementary Fig. 7). Inactivation of PLK3 has demonstrated to induce G2/M arrest (Wang et al. 2002).

Volasertib decreased cells in the S phase in BHP7-13, K1, FTC-133 and RO82-W-1 cells, which is likely another therapeutic mechanism of volasertib in WDTC cell lines. A failure of entry from G1 to $S$ phase may account for the decreased cells in S phase. PLK2 and PLK3 are pivotal for the process of G1/S transition. PLK2 is required for centriole duplication and onset of $S$ phase (Cizmecioglu et al. 2012). Depletion of PLK2 leads to delayed entry into the $\mathrm{S}$ phase (Ma et al. 2003). PLK3 expression peaks in the
G1 phase, and inhibition of PLK3 results in prevention of the $S$ phase entry (Zimmerman \& Erikson 2007). The ability of volasertib to inhibit PLK2 and PLK3 may contribute to the failure of $\mathrm{G} 1 / \mathrm{S}$ transition and subsequently decrease cells in the $S$ phase in thyroid cancer cells. Another explanation for the decreased cells in the $S$ phase may be as a result of fewer cells entering the G1 phase because of G2/M arrest.

Tumor cells often escape apoptosis, and induction of apoptosis is considered an important mechanism of anticancer therapies. PLK1 is involved in the regulation of apoptosis; therefore, inhibition of PLK1 activity is likely to contribute to the apoptotic response (Liu \& Erikson 2003). Volasertib treatment activated caspase-3 activity 
and induced apoptosis in WDTC cell lines, which is likely one of the mechanisms of cytotoxicity in the treatment of thyroid cancer.

RO82-W-1 cells were the least sensitive cell lines to volasertib treatment in terms of the highest $\mathrm{IC}_{50}$ among 4 WDTC cell lines. RO82-W-1 cells were more resistant to sub-G1 apoptosis induced by volasertib treatment. In addition, RO82-W-1 cells had a relatively slower cell proliferation rate (Supplementary Fig. 8). Slow growing cells are likely to be less sensitive to cell cycle inhibition as compared with rapidly growing cells. These observations may account for the lower sensitivity of RO82-W-1 cells to volasertib therapy.

Volasertib treatment significantly repressed K1 tumor growth. The anti-tumor effects are likely mediated through both cell cycle inhibition and apoptosis induction given that PCNA levels decreased and cleaved caspase-3 levels increased with volasertib treatment in K1 xenografts.

Sorafenib therapy is an US FDA-approved treatment for WDTC. However, many patients with refractory WDTC stop sorafenib treatment because of side effects or treatment failures. Devising novel strategies to augment the therapeutic effects of sorafenib is essential. In this study, we found promising therapeutic effects of the volasertib and sorafenib combination therapy in the treatment of FTC-133 tumors. This combination therapy inhibited FTC-133 tumor growth significantly as compared with either drug treatment alone with favorable safety profiles.

To explore potential biomarkers that correlate with the sensitivity of volasertib, the baseline expressions of PLK1, PLK2 and PLK3 were evaluated in four WDTC cell lines (Supplementary Fig. 9A). The sensitivity of these cell lines to volasertib was ordered according to the $\mathrm{IC}_{50}$ value. Statistical relationships were analyzed using Pearson's correlation coefficient (Supplementary Fig. 9B). The expressions of PLK1, PLK2 and PLK3 failed to show any significant correlation with volasertib sensitivity in WDTC cells.

We found volasertib treatment led to increased PLK1 expression in WDTC in vitro and in vivo. The reasons accounting for this phenomenon are unclear. One tentative explanation is that PLK1 expression was increased to compensate the decreased activity of PLK1.

A recent report reveals that the combination of volasertib and PI3K inhibitors was synergistic in the treatment of anaplastic thyroid cancer (ATC) cells in vitro (De Martino et al. 2018). Combining volasertib and a PI3K inhibitor was more effective in inhibiting ATC tumor growth than either single agent therapy and placebo

C) 2019 Society for Endocrinology Published by Bioscientifica Ltd. Printed in Great Britain treatment. These findings are encouraging and potentially broaden the clinical applications of volasertib in the treatment of thyroid cancer. Besides, many PLK inhibitors are under clinical evaluation in the treatment of cancers (ClinicalTrials.gov, accessed April 11, 2019).

RO82-W-1 and WRO82-1 are considered to be the same cell line with different designations (SIB Bioinformatics Resource Portal, accessed May 24, 2019). However, we found the DNA STR profiling of RO82-W-1 cells was different to that of WRO82-1 cells (Supplementary Fig. 10, Schweppe et al. 2008). We acknowledge RO82-W-1 is not the same as WRO82-1. Besides, our data reveal that RO82-W-1 cells had WT BRAF V600.

\section{Conclusions}

Volasertib induces cytotoxicity in both papillary and follicular thyroid cancer cell lines. The therapeutic efficacy and safety of volasertib treatment were demonstrated in a xenograft mouse model. Volasertib and sorafenib combination therapy exhibited greater therapeutic efficacy over either single treatment in FTC-133 tumors. These data support the clinical evaluation of volasertib in the treatment of patients with WDTC.

\section{Supplementary data}

This is linked to the online version of the paper at https://doi.org/10.1530/ ERC-18-0555.

\section{Declaration of interest}

The authors declare that there is no conflict of interest that could be perceived as prejudicing the impartiality of the research reported.

\section{Funding}

This work was supported by the Chang Gung Memorial Hospital (CMRPG3H0201, CMRPG3E0353) and the Ministry of Science and Technology of Taiwan (MOST 106-2314-B-182A-095).

\section{Author contribution statement}

S-F L and C-N Y conceived the work; S-F L and R W designed the study; J-D L, T-C C and R W provided material support; S-F L acquired the data; all authors analyzed and interpreted the data; S-F L wrote the manuscript and all authors reviewed and revised the manuscript.

\section{Acknowledgments}

The authors thank the staff of the Microscope Core Laboratory, the Laboratory Animal Center and the Expensive Advanced Instrument Core Laboratory of the Chang Gung Memorial Hospital at Linkou for technical support. 


\section{References}

Brose MS, Nutting CM, Jarzab B, Elisei R, Siena S, Bastholt L, de la Fouchardiere C, Pacini F, Paschke R, Shong YK, et al. 2014 Sorafenib in radioactive iodine-refractory, locally advanced or metastatic differentiated thyroid cancer: a randomised, double-blind, phase 3 trial. Lancet 384 319-328. (https://doi.org/10.1016/S01406736(14)60421-9)

Cabanillas ME, McFadden DG \& Durante C 2016 Thyroid cancer. Lancet 388 2783-2795. (https://doi.org/10.1016/S0140-6736(16)30172-6)

Chou TC 2006 Theoretical basis, experimental design, and computerized simulation of synergism and antagonism in drug combination studies. Pharmacological Reviews $\mathbf{5 8}$ 621-681. (https://doi. org/10.1124/pr.58.3.10)

Chou TC \& Martin N 2005 CompuSyn for Drug Combinations: PC Software and Users Guide: A Computer Program for Quantitation of Synergism and Antagonism in Drug Combinations and the Determination of IC50, ED50, and LD50 Values. Paramus, NJ, USA: ComboSyn. (available at: http://www.combosyn.com/)

Cizmecioglu O, Krause A, Bahtz R, Ehret L, Malek N \& Hoffmann I 2012 Plk2 regulates centriole duplication through phosphorylationmediated degradation of Fbxw7 (human Cdc4). Journal of Cell Science 125 981-992. (https://doi.org/10.1242/jcs.095075)

de Cárcer G, Manning G \& Malumbres M 2011a From Plk1 to Plk5: functional evolution of polo-like kinases. Cell Cycle 10 2255-2262. (https://doi.org/10.4161/cc.10.14.16494)

de Cárcer G, Escobar B, Higuero AM, García L, Ansón A, Pérez G, Mollejo M, Manning G, Meléndez B, Abad-Rodríguez J, et al. 2011b Plk5, a polo box domain-only protein with specific roles in neuron differentiation and glioblastoma suppression. Molecular and Cellular Biology 31 1225-1239. (https://doi.org/10.1128/MCB.00607-10)

De Martino D, Yilmaz E, Orlacchio A, Ranieri M, Zhao K \& Di Cristofano A 2018 PI3K blockage synergizes with PLK1 inhibition preventing endoreduplication and enhancing apoptosis in anaplastic thyroid cancer. Cancer Letters 439 56-65. (https://doi.org/10.1016/j. canlet.2018.09.024)

Durante C, Haddy N, Baudin E, Leboulleux S, Hartl D, Travagli JP, Caillou B, Ricard M, Lumbroso JD, De Vathaire F, et al. 2006 Longterm outcome of 444 patients with distant metastases from papillary and follicular thyroid carcinoma: benefits and limits of radioiodine therapy. Journal of Clinical Endocrinology and Metabolism 91 2892-2899. (https://doi.org/10.1210/jc.2005-2838)

Fagin JA \& Wells SA Jr 2016 Biologic and clinical perspectives on thyroid cancer. New England Journal of Medicine 375 1054-1067. (https://doi.org/10.1056/NEJMra1501993)

Gjertsen BT \& Schöffski P 2015 Discovery and development of the Pololike kinase inhibitor volasertib in cancer therapy. Leukemia 29 11-19. (https://doi.org/10.1038/leu.2014.222)

Habedanck R, Stierhof YD, Wilkinson CJ \& Nigg EA 2005 The Polo kinase Plk4 functions in centriole duplication. Nature Cell Biology $\mathbf{7}$ 1140-1146. (https://doi.org/10.1038/ncb1320)

Helmke C, Becker S \& Strebhardt K 2016 The role of Plk3 in oncogenesis. Oncogene 35 135-147. (https://doi.org/10.1038/ onc.2015.105)

Kitahara CM \& Sosa JA 2016 The changing incidence of thyroid cancer. Nature Reviews: Endocrinology 12 646-653. (https://doi.org/10.1038/ nrendo.2016.110)

Kosco K, Ridinger M, Whitley P, Croucher P, Miner JN \& Erlander M 2018 Abstract 1885: Selective Polo-like kinase 1 (PLK1) inhibitor PCM-075 is highly active alone and shows synergy when combined with FLT3 inhibitors in models of acute myeloid leukemia (AML). Cancer Research 78 (13 Supplement) abstract 1885. (https://doi. org/10.1158/1538-7445.AM2018-1885)

Lin SF, Lin JD, Hsueh C, Chou TC \& Wong RJ 2017 A cyclin-dependent kinase inhibitor, dinaciclib in preclinical treatment models of thyroid cancer. PLoS ONE 12 e0172315. (https://doi.org/10.1371/ journal.pone.0172315)

Lin SF, Lin JD, Hsueh C, Chou TC \& Wong RJ 2018 Potent effects of roniciclib alone and with sorafenib against well-differentiated thyroid cancer. Endocrine-Related Cancer 25 853-864. (https://doi. org/10.1530/ERC-18-0150)

Liu X \& Erikson RL 2003 Polo-like kinase (Plk)1 depletion induces apoptosis in cancer cells. PNAS 100 5789-5794. (https://doi. org/10.1073/pnas.1031523100)

Ma S, Charron J \& Erikson RL 2003 Role of Plk2 (Snk) in mouse development and cell proliferation. Molecular and Cellular Biology 23 6936-6943. (https://doi.org/10.1128/mcb.23.19.6936-6943.2003)

Moldovan GL, Pfander B \& Jentsch S 2007 PCNA, the maestro of the replication fork. Cell 129 665-679. (https://doi.org/10.1016/j. cell.2007.05.003)

Nguyen T, Parker R, Hawkins E, Holkova B, Yazbeck V, Kolluri A, Kmieciak M, Rahmani M \& Grant S 2017 Synergistic interactions between PLK1 and HDAC inhibitors in non-Hodgkin's lymphoma cells occur in vitro and in vivo and proceed through multiple mechanisms. Oncotarget 8 31478-31493. (https://doi.org/10.18632/ oncotarget.15649)

Pfeffer CM \& Singh ATK 2018 Apoptosis: a target for anticancer therapy. International Journal of Molecular Sciences 19 448. (https://doi. org/10.3390/ijms19020448)

Pujade-Lauraine E, Selle F, Weber B, Ray-Coquard IL, Vergote I, Sufliarsky J, Del Campo JM, Lortholary A, Lesoin A, Follana P, et al. 2016 Volasertib versus chemotherapy in platinum-resistant or -refractory ovarian cancer: a randomized phase II Groupe des Investigateurs Nationaux pour l'Etude des Cancers de l'Ovaire study. Journal of Clinical Oncology 34 706-713. (https://doi.org/10.1200/ JCO.2015.62.1474)

Raab M, Krämer A, Hehlgans S, Sanhaji M, Kurunci-Csacsko E, Dötsch C, Bug G, Ottmann O, Becker S, Pachl F, et al. 2015 Mitotic arrest and slippage induced by pharmacological inhibition of Polo-like kinase 1. Molecular Oncology 9 140-154. (https://doi.org/10.1016/j. molonc.2014.07.020)

Rudolph D, Steegmaier M, Hoffmann M, Grauert M, Baum A, Quant J, Haslinger C, Garin-Chesa P \& Adolf GR 2009 BI 6727, a Polo-like kinase inhibitor with improved pharmacokinetic profile and broad antitumor activity. Clinical Cancer Research 15 3094-3102. (https:// doi.org/10.1158/1078-0432.CCR-08-2445)

Rudolph D, Impagnatiello MA, Blaukopf C, Sommer C, Gerlich DW, Roth M, Tontsch-Grunt U, Wernitznig A, Savarese F, Hofmann MH, et al. 2015 Efficacy and mechanism of action of volasertib, a potent and selective inhibitor of Polo-like kinases, in preclinical models of acute myeloid leukemia. Journal of Pharmacology and Experimental Therapeutics 352 579-589. (https://doi.org/10.1124/jpet.114.221150)

Schlumberger M, Tahara M, Wirth LJ, Robinson B, Brose MS, Elisei R, Habra MA, Newbold K, Shah MH, Hoff AO, et al. 2015 Lenvatinib versus placebo in radioiodine-refractory thyroid cancer. New England Journal of Medicine 372 621-630. (https://doi.org/10.1056/ NEJMoa1406470)

Schweppe RE, Klopper JP, Korch C, Pugazhenthi U, Benezra M, Knauf JA, Fagin JA, Marlow LA, Copland JA, Smallridge RC, et al. 2008 Deoxyribonucleic acid profiling analysis of 40 human thyroid cancer cell lines reveals cross-contamination resulting in cell line redundancy and misidentification. Journal of Clinical Endocrinology and Metabolism 93 4331-4341. (https://doi.org/10.1210/jc.20081102)

Sebastian M, Reck M, Waller CF, Kortsik C, Frickhofen N, Schuler M, Fritsch H, Gaschler-Markefski B, Hanft G, Munzert G, et al. 2010 The efficacy and safety of BI 2536, a novel Plk-1 inhibitor, in patients with stage IIIB/IV non-small cell lung cancer who had relapsed after, or failed, chemotherapy: results from an open-label, randomized phase II clinical trial. Journal of Thoracic Oncology 5 1060-1067. (https://doi.org/10.1097/JTO.0b013e3181d95dd4)
(C) 2019 Society for Endocrinology Published by Bioscientifica Ltd. Printed in Great Britain 
Shao C, Chien SJ, Farah E, Li Z, Ahmad N \& Liu X 2018 Plk1 phosphorylation of Numb leads to impaired DNA damage response. Oncogene 37 810-820. (https://doi.org/10.1038/onc.2017.379)

Strebhardt K 2010 Multifaceted polo-like kinases: drug targets and antitargets for cancer therapy. Nature Reviews: Drug Discovery 9 643-660. (https://doi.org/10.1038/nrd3184)

Strebhardt K \& Ullrich A 2006 Targeting polo-like kinase 1 for cancer therapy. Nature Reviews: Cancer 6 321-330. (https://doi.org/10.1038/ nrc1841)

Wang Q, Xie S, Chen J, Fukasawa K, Naik U, Traganos F, Darzynkiewicz Z, Jhanwar-Uniyal M \& Dai W 2002 Cell cycle arrest and apoptosis induced by human polo-like kinase 3 is mediated through perturbation of microtubule integrity. Molecular and Cellular
Biology 22 3450-3459. (https://doi.org/10.1128/mcb.22.10.34503459.2002)

Yata K, Lloyd J, Maslen S, Bleuyard JY, Skehel M, Smerdon SJ \& Esashi F 2012 Plk1 and CK2 act in concert to regulate Rad51 during DNA double strand break repair. Molecular Cell 45 371-383. (https://doi. org/10.1016/j.molcel.2011.12.028)

Zimmerman WC \& Erikson RL 2007 Polo-like kinase 3 is required for entry into S phase. PNAS 104 1847-1852. (https://doi.org/10.1073/ pnas.0610856104)

Zitouni S, Nabais C, Jana SC, Guerrero A \& Bettencourt-Dias M 2014 Polo-like kinases: structural variations lead to multiple functions. Nature Reviews: Molecular Cell Biology 15 433-452. (https://doi. org/10.1038/nrm3819)

Received in final form 28 May 2019

Accepted 11 June 2019

Accepted Preprint published online 11 June 2019 (c) 2019 Society for Endocrinology Published by Bioscientifica Ltd. Printed in Great Britain 